\title{
Endomyocardial Fibrosis Associated with Massive Calcification of the Left Ventricle
}

\author{
Manoel Fernandes Canesin, Renato Faria da Gama, Débora Lee Smith, Flávio Jun Kazuma, \\ Arlei Takiuchi, Antonio Carlos Pereira Barretto
}

Londrina, PR - São Paulo, SP - Brazil

\begin{abstract}
This is the report of a rare case of endomyocardial fibrosis associated with massive calcification of the left ventricle in a male patient with dyspnea on great exertion, which began 5 years earlier and rapidly evolved. Due to lack of information and the absence of clinical signs that could characterize impairment of other organs, the case was initially managed as a disease with a pulmonary origin. With the evolution of the disease and in the presence of radiological images of heterogeneous opacification in the projection of the left ventricle, the diagnostic hypothesis of endomyocardial disease was established. This hypothesis was later confirmed on chest computed tomography. The patient died on the $16^{\text {th }}$ day of the hospital stay, probably because of lack of myocardial reserve, with clinical findings of refractory heart failure, possibly aggravated by pulmonary infection. This shows that a rare disease such as endomyocardial fibrosis associated with massive calcification of the left ventricle may be suspected on a simple chest $X$-ray and confirmed by computed tomography.
\end{abstract}

Endomyocardial fibrosis is a rare disease, but in Brazil and other tropical and subtropical countries its prevalence has been increasing ${ }^{1}$. It is characterized by fibrosis of the endocardium and myocardium, and of the inlet and apex of either the right or left or both ventricles. Most of the time, its clinical evolution is progressive with findings of refractory heart failure.

We report a case of difficult diagnosis due to its discreet initial clinical manifestation, and also rare due to its association with massive endomyocardial calcification. Initially, the diagnostic hypothesis of primary pulmonary disease was formulated, and the investigation followed that direction. Later, based on radiological and tomographic images of calcification in the cardiac area, the hypothesis of endomyocardial fibrosis was formulated.

Hospital Universitário da Universidade Estadual de Londrina and Instituto do Coração do Hospital das Clínicas - FMUSP

Mailing address: Manoel Fernandes Canesin - Rua Dr. Elias Cesar, 155/701 86015-640 - Londrina, PR, Brazil.

Submitted: 04/01/99

Accepted: 06/16/99

\section{Report of the case}

The patient is a 23 -year-old white male, $1.73 \mathrm{~m}$ tall and weighing $60 \mathrm{~kg}$, who sought medical treatment complaining of progressive dyspnea. The patient reported that at the age of 18 years, dyspnea on great exertion began and evolved with paroxysmal periods of worsening and improvement. One month prior to hospital presentation, the dyspnea evolved to dyspnea on medium and mild exertion. He also reported having repetitive episodes of pulmonary infection since childhood. He denied orthopnea, paroxysmal nocturnal dyspnea, and chest pain. He experienced sporadic diarrheas prior to hospital admission. He denied fever. The patient reported the presence of a painless precordial prominence since the age of 16 years, and also a familial antecedent of coronary heart disease. He denied smoking or having other associated diseases.

On physical examination, the patient was in regular condition, with peripheral cyanosis and tachypnea. The precordial prominence referred to in the history was evident, with no local inflammatory signs or evidence of bone deformity. Blood pressure was $130 / 80 \mathrm{mmHg}$ and the heart rate was $90 \mathrm{bpm}$. Cardiac rhythm was regular with normal cardiac sounds and no murmurs. The lungs had disseminated crepitant rales and sibili bilaterally. The remaining findings of the physical examination were within the normal range.

Spirometry revealed a severe restrictive disorder. During the first days at the hospital, the patient developed fever with a dry cough and worsening of the dyspnea. Treatment with bronchodilators and antibiotics was started.

Blood and urine tests were within the normal range. The following tests were negative: anti-HIV, tubercle bacilli, and fungus in the sputum. Restelectrocardiogram showed leftbundlebranch block, with deviation of the electrical axis to the left, and right ventricle overload. On chest X-ray, lungs with moderate congestion were evidenced, as was an enlargement of the cardiac silhouette (+/4+), with animage of heterogeneous opacificationin the projection of the left ventricle(Fig. 1).Computed tomography of the chest showed condensation of some foci located in the pulmonary parenchyma, enlargement in cardiac volume, and intracardiacimages of massive calcification(Fig.2).

Transthoracic and transesophageal echocardiograms were performed with technical difficulties. The transthora- 


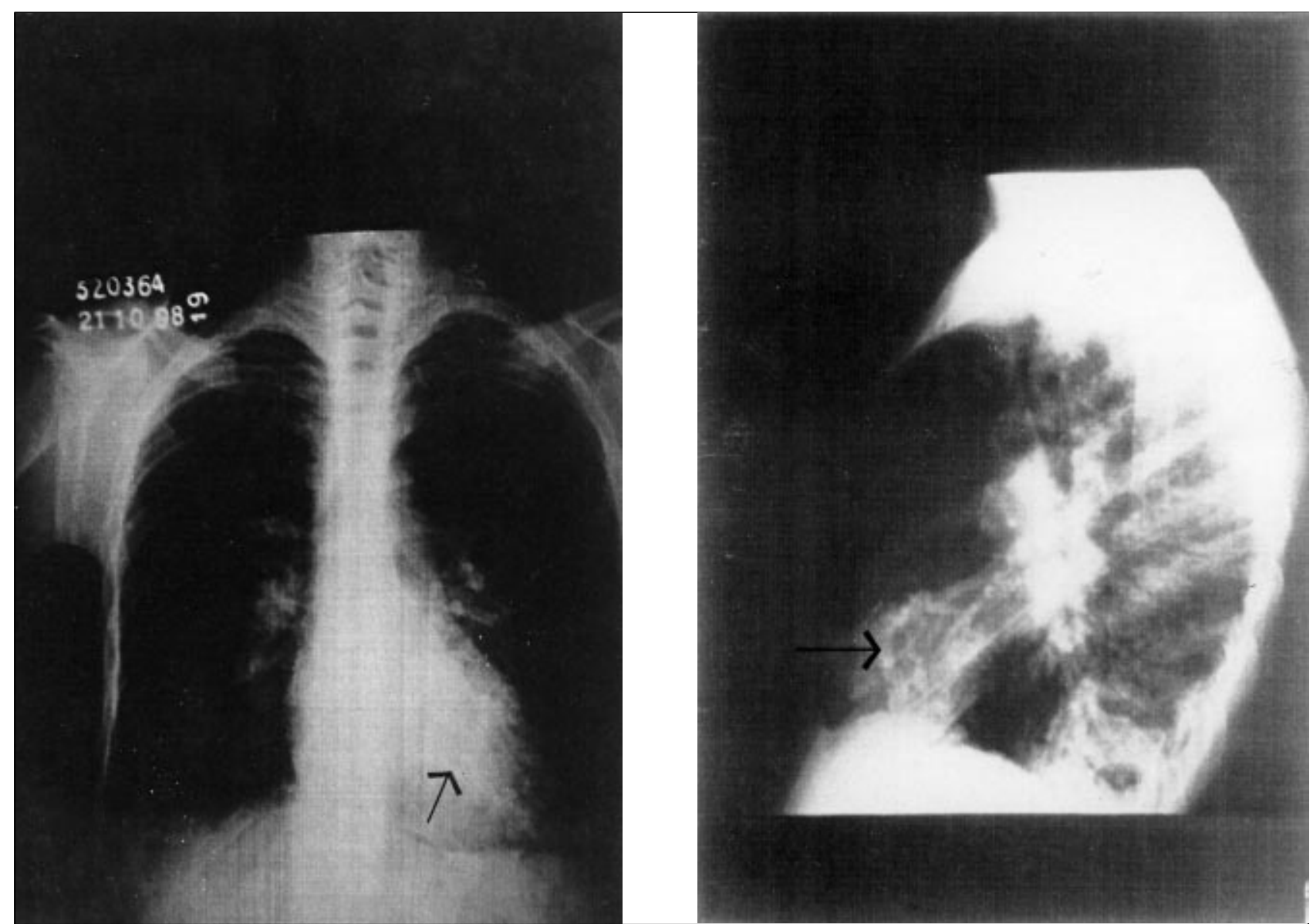

Fig. 1-A) Chest X-ray (posteroanterior view) showing heterogeneous opacification in the projection of the LV; B) Chest X-ray (lateral view) showing heterogeneous opacification in the projection of the LV.

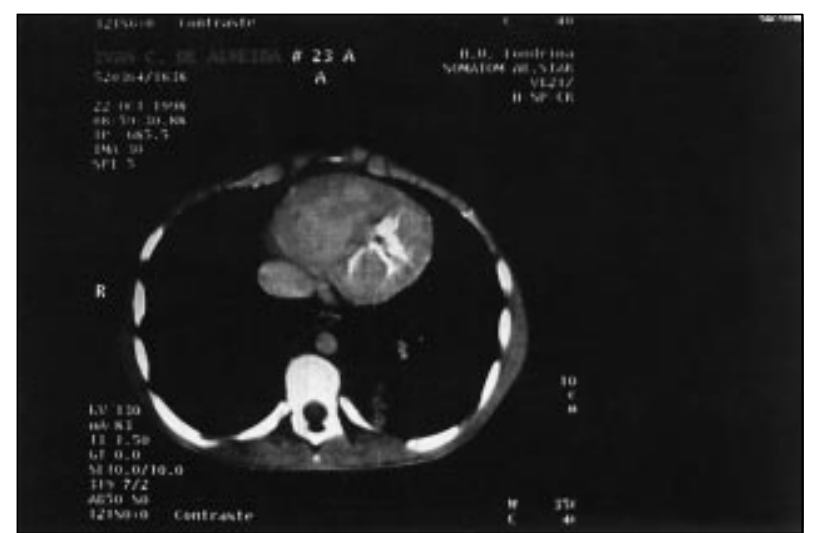

Fig. 2 - Chest computed tomography showing intracardiac image of massive calcification.

cic echocardiogram was inconclusive due to lack of a thoracic window for performing the test. The transesophageal echocardiogram was also inconclusive because of the patient's intolerance due to dyspnea and nausea at the time of the test. However, mild thickening of the septum was evidenced, with mild hypokinesia of the left ventricle. Left ventricle ejection fraction could not be quantified. Tricuspid regurgitation and pulmonary arterial hypertension with an increase in the right ventricle volume were observed.
The patient's condition significantly worsened. He was transferred to the intensive care unit and died on the $16^{\text {th }}$ day of the hospital stay.

On autopsy, the gross examination showed fibrosis and massive calcification of the left ventricle, particularly at the apex, spreading to the left ventricle outflow tract (Fig. 3). Microscopy confirmed the presence of significant fibrosis covering a large area of the endocardium (Fig. 4). The radio-

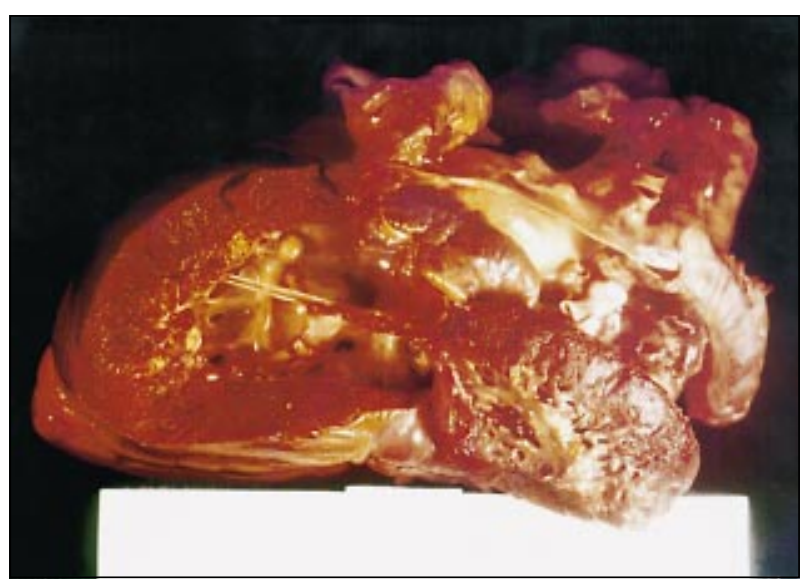

Fig.3-Anatomicopathological examination showing, grossly, massive calcification of the left ventricle (predominating in the apex but extending until the outflow tract of the LV). 


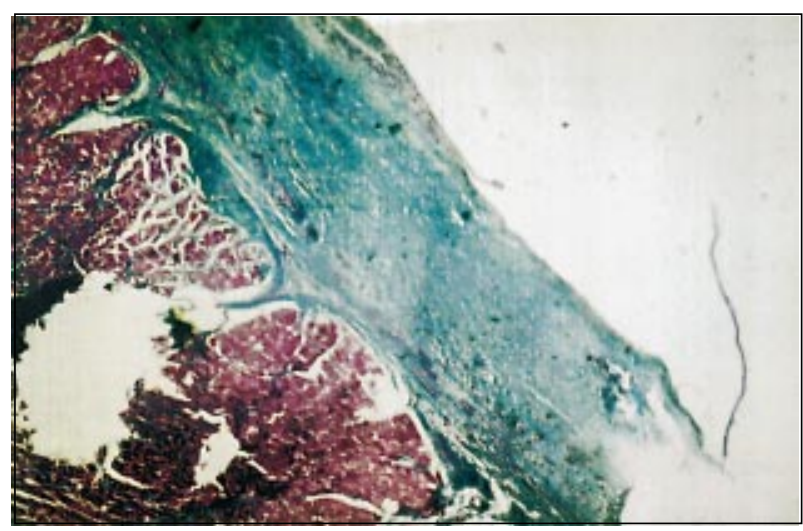

Fig. 4 - Histopathology of the endomyocardium with significant fibrosis and calcification

logical image obtained after the autopsy (Fig. 5) showed in greater detail the intense calcification of the heart.

\section{Discussion}

Conditions leading to impairment of left ventricle filling are various, endomyocardial fibrosis being one of them ${ }^{1}$. Endomyocardial fibrosis is an infrequent disease, more prevalent in tropical countries. Its clinical findings

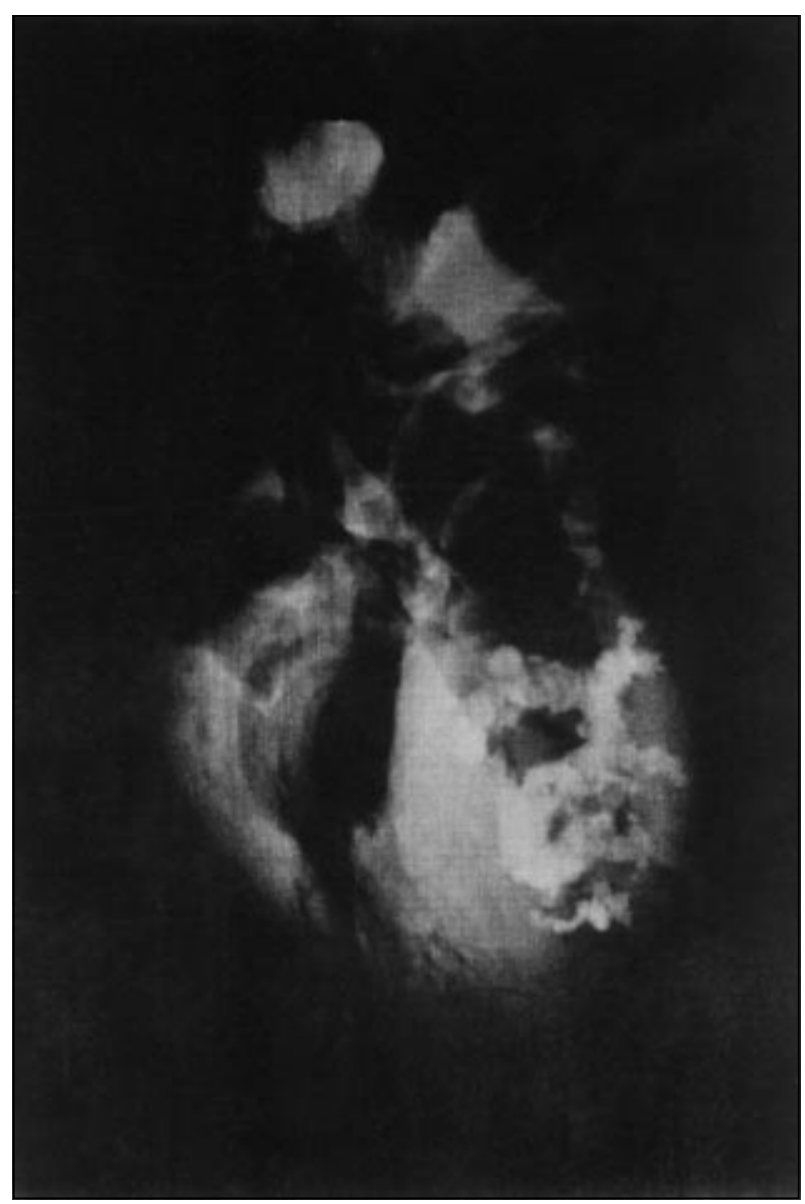

Fig. 5 - Chest computed tomography showing intracardiac image of massive calcification. depend on the cardiac chambers affected and on their degree of involvement. Precordial pain suggests a predominant involvement of the left ventricle; ascites, pericardial effusion and a low prothrombin time suggest a predominant impairment of the right ventricle. Dyspnea and edema are common findings in the involvement of both ventricles, and it is difficult to differentiate which ventricle is more affected based on these data ${ }^{2}$. Involvement of both ventricles, right ventricle fibrosis, and tricuspid or mitral regurgitation are associated with a higher mortality ${ }^{3}$. Endocardial calcification is described in some case series but is not a frequent finding ${ }^{4-6}$.

Endomyocardial fibrosis is a disease of unknown etiology, first described in Brazil in 19547 . In 1984, Silver et al ${ }^{1}$ described the first case of massive endocardial calcification of the left ventricle, suggesting it was a different entity causing restrictive cardiomyopathy. This suggestion was refuted by Lengyel et $\mathrm{al}^{8}$, who suggested that the endocardial calcification was a clue for the diagnosis of endomyocardial fibrosis.

In our patient's clinical history, complaints of dyspnea and wheezing predominated, with previous repetitive respiratory infections. In addition, fever during hospital stay and absence of cardiac murmur or gallop rhythm suggested that the disease was primarily of pulmonary origin. A simple chest X-ray was performed showing a heterogeneous opacification in the left ventricle projection, with a mild increase in the cardiac silhouette and pulmonary congestion. Later, a computed tomography was performed, showing an image of significant calcification in the left ventricle.

After these results, we began to consider the possibility of primary heart disease and started treatment with a diuretic, which resulted in a significant improvement in symptoms. An echocardiogram was also performed, with little help for the diagnosis, showing only pulmonary hypertension and tricuspid regurgitation.

In a retrospective analysis, the first evidence of heart disease was provided by the chest X-ray, because more specific tests, such as transthoracic and transesophageal echocardiograms were technically impaired. The left ventricle calcification present in the simple X-ray and later confirmed on the chest computed tomography was the great sign pointing to the diagnosis of endomyocardial fibrosis with massive calcification of the left ventricle. The rapid evolution to death was attributed to a pulmonary infection, which exhausted the patient's small myocardial reserve. Endomyocardial fibrosis could have been diagnosed by ventriculography, whose image is considered the gold standard for the diagnosis of that disease. The patient, however, died before undergoing that test. Therefore, the findings of the chest computed tomography made the diagnosis possible. Later, the anatomicopathological study confirmed the diagnosis of endomyocardial fibrosis.

The tomographic images obtained in the present case were very similar to those presented by Lengyel et $a^{8}$, and their aspects were very characteristic not allowing many options of differential diagnosis besides endomyocardial 
fibrosis with calcification. In addition, the density of the image of the massive calcium deposit, even denser than the patient's own body of vertebra, indicates the diagnosis. Its finding, even though uncommon, helps in the clinical charcterization of the disease, allowing the identification of the most affected chamber ${ }^{2}$. Massive calcification, as in this case, is even rarer.

It is worth emphasizing that even with the massive calcification here presented, the patient remained with only a few symptoms for a long period of time, worsening more intensely as death approached. Another peculiarity of the case is the anatomicopathological finding of marked fibrosis with calcification spreading to the outflow tract of the left ventricle, which is also rare in patients with endomyocardial fibrosis.

These data show that a simple chest X-ray may direct the diagnosis of endomyocardial fibrosis with calcification. Later, the chest computed tomography confirmed the presence of massive cardiac calcification in a very characteristic way. The massive and dense intracardiac calcification revealed by chest computed tomography may be pathognomonical or, at least, very suggestive of endomyocardial fibrosis. Finally, the anatomicopathological findings confirmed the diagnosis of endomyocardial fibrosis with massive calcification of the left ventricle.

\section{References}

1. Silver MA, Bonow RO, Deglin SM, et al. Acquired left ventricular endocardial constriction for massive mural calcific deposits: a newly recognized cause of inpairment to left ventricular filling. Am J Cardiol 1984; 53: 1468-70.

2. Barretto ACP, Mady C, Arteaga E, et al. Quadro clínico da endomiocardiofibrose. Correlação com a intensidade da fibrose. Arq Bras Cardiol 1988; 51: 401-5.

3. Barretto ACP, Luz PL, Oliveira AS, et al. Determinants of survival in endomyocardial fibrosis. Circulation 1989; 80: (suppl. I): I-177 - I-182.

4. Cockshott, WP, Saric S, Ikeme AC. Radiological findings in endomyocardial fibrosis. Circulation 1967; 35: 913-22.
5. Fernandes F, Mady C, Vianna CB, et al. Aspectos radiológicos da endomiocardiofibrose. Arq Bras Cardiol 1996; 67: 103-5.

6. Morrone LFP, Moreira ELC, Lopez HM. Endomiocardiofibrose com calcificação endocárdica maciça biventricular. Arq Bras Cardiol 1996; 67: 103-5

7. Ball JD, Williams AW, Davies JNP. Endomyocardial fibrosis. Lancet 1954; 1: 1049 .

8. Lengyel M, Árvay A, Palik I, et al. Massive endocardial calcification associated endomyocardial fibrosis. Am J Cardiol 1985; 56: 815-6. 